\title{
Surgical treatment of gastrointestinal stromal tumours combined with imatinib treatment: a retrospective cohort analysis
}

\author{
Robert Bachmann, Jens Strohäker, Julian Kraume, Alfred Königsrainer, Ruth Ladurner \\ Department of General, Visceral and Transplant Surgery, University Hospital Tübingen, Tübingen, Germany \\ Contributions: (I) Conception and design: R Bachmann; (II) Administrative support: J Strohäker; (III) Provision of study materials or patients: R \\ Bachmann, R Ladurner, A Königsrainer; (IV) Collection and assembly of data: J Strohäker, J Kraume; (V) Data analysis and interpretation: R \\ Bachmann, R Ladurner; (VI) Manuscript writing: All authors; (VII) Final approval of manuscript: All authors. \\ Correspondence to: Robert Bachmann, MD. Department of Surgery, University Hospital Tübingen, Tübingen, Germany. \\ Email: robert.bachmann@med.uni-tuebingen.de.
}

\begin{abstract}
Background: Targeted therapies changed the treatment concepts of gastrointestinal stromal tumours significantly. As only possibility to cure surgical resection is the cornerstone of therapy. Thus it is necessary to find out which patients will benefit most regarding modality (neo- or adjuvant) and duration of chemotherapy.

Methods: In a retrospective cohort analysis the medical records of all consecutive patients treated in the department of general and visceral surgery of the university hospital Tübingen between 2004 and 2015 were investigated. Recurrence and survival outcomes were calculated using the Kaplan-Meier method.

Results: Tumor location of GIST was gastric in 32, small bowel in 14, rectum in 3 and extraintestinal in 3 patients. Median tumor size was $46 \mathrm{~mm}$. Median mitotic index was 4 per 50 hpf. Resection was achieved R0 in 46 patients, R1 in 4 patients and R2 in 2 patients. Mean overall survival was 58.9 months (range, 4673 months). Mean recurrence free survival was 45.6 months (range, 36-57 months). Mean overall survival was 58.9 months (range, 46-73 months). Risk factors for recurrence were tumor location and high mitotic index $\mathrm{Ki}-67$.
\end{abstract}

Conclusions: The prognosis of GIST after surgical resection is favourable. Survival prognosis are excellent. Analysis of KI-67 mitotic index predicted best oncologic outcome.

Keywords: Gastrointestinal stromal tumors surgery (GIST surgery); Ki-67 in GIST; oncologic outcome

Received: 23 November 2018; Accepted: 30 November 2018; Published: 27 December 2018.

doi: $10.21037 / \operatorname{tgh} .2018 .12 .02$

View this article at: http://dx.doi.org/10.21037/tgh.2018.12.02

\section{Introduction}

Gastrointestinal stromal tumors (GIST) are the most common gastrointestinal mesenchymal tumors (1).

Pathophysiologically, activating mutations of KIT or platelet derived growth factor receptor alpha (PDGFRA) gene are leading to continuous tyrosine kinase activity and uncontrolled cell proliferation (2).

Main clinicopathological parameters of GIST's malignancy are dependant of tumor size and location, number of mitoses and distant tumor spread. Complete surgical resection within tumour-free margins is standard surgical therapy. As lymph nodal involvement is seldom, lymphadenectomy is not generally recommended (3). High rates of local or distant recurrences of about $40 \%$ required the introduction of imatinib mesylate for a duration of 3 years as standard adjuvant therapy after surgical resection of non-metastatic GISTs with significant risk of recurrence (4). Neoadjuvant imatinib is indicated in locally advanced or unresectable GIST to downsize the tumor and to reduce the extent of resection.

As GISTs are heterogeneous and not entirely understood actual treatment concepts are being further revised. Herein, we present the investigation of clinicopathologic characteristics of patients with primary GIST in a retrospective cohort study. Aim of our study was to analyze 
Table 1 Baseline demographics

\begin{tabular}{|c|c|}
\hline Parameter & $\mathrm{n}(\%)$ \\
\hline Median age (range) years & $61.5[24-87]$ \\
\hline Sex, male (\%) & 59.6 \\
\hline Symptoms & $27(51.9)$ \\
\hline Median tumor size, mm (range) & 48 [4-295] \\
\hline$<10$ & $4(7.7)$ \\
\hline 31 & $5(9.6)$ \\
\hline 71 & $20(38.5)$ \\
\hline 151 & $15(28.8)$ \\
\hline 251 & $4(7.7)$ \\
\hline$>151$ & $4(7.7)$ \\
\hline \multicolumn{2}{|c|}{ GIST ( $n=52)$ mitotic count (per 50 HPF) } \\
\hline$\leq 5$ & $35(67.3)$ \\
\hline $6-10$ & $7(13.5)$ \\
\hline$>10$ & $8(15.4)$ \\
\hline Unknown & $2(3.8)$ \\
\hline \multicolumn{2}{|l|}{ Exact location } \\
\hline Stomach & $32(61.5)$ \\
\hline Duodenum & $3(5.8)$ \\
\hline Small bowel & $12(23.1)$ \\
\hline Colon & 0 \\
\hline Rectum & $3(5.8)$ \\
\hline Extragastrointestinal & $3(5.8)$ \\
\hline \multicolumn{2}{|l|}{ NIH risk (GIST) $n=52$} \\
\hline Unknown & $2(3.8)$ \\
\hline Benign & $5(9.6)$ \\
\hline Very low & $9(17.3)$ \\
\hline Low & $13(25.0)$ \\
\hline Intermediate & $10(19.2)$ \\
\hline High & $13(25.0)$ \\
\hline
\end{tabular}

GIST, gastrointestinal stromal tumors; $\mathrm{NIH}$, National Institutes of Health.

outcome of surgery and targeted chemotherapy according to known prognostic factors.

\section{Methods}

\section{Patients and study design}

The study was performed as a single center evaluation in the sarcoma center of the University of Tübingen. The study included all consecutive patients with primary GIST that had surgical resection in the time period between 2004 until 2015. All consecutive patients were retrospectively evaluated. The diagnosis of GIST was established by pathohistology and immunohistochemistry. Analyses included patient demographics, tumor location, classification of grading, surgical treatment, surgical outcome and oncological outcome. Follow-up was conducted using the standardized medical records. Disease free survival was defined as the period from surgery to recurrence or end of follow-up.

\section{Endpoints}

Primary endpoints were overall survival and disease or recurrence free survival.

Secondary endpoint was time of adjuvant imatinib and recurrence free survival.

\section{Statistical analyses}

Statistical analysis was done using SPSS software, version 21 (SPSS Inc., Chicago, IL, USA). Results are reported as median and lower and upper quartiles. Survival curves were calculated using Kaplan-Meier analysis and the log rank test. For testing significant differences between the examined groups, Student's $t$-test and the Mann-Whitney $\mathrm{U}$ test was used. A significance level $<0.05$ was defined.

\section{Results}

\section{Baseline demographics and clinical characteristics}

Fifty-two patients were operated during the study period. Median age was 61.5 years. Male to female ratio was 31 to $21(59.6 \%$ male). Tumor location of GIST was stomach in 32 patients $(61.5 \%)$, small intestine in $14(26.9 \%)$, colon in 0 , rectum in $3(5.8 \%)$ and extraintestinal in 3 patients (5.8\%). Preoperative biopsy was performed in $33 \%$ of patients. Median tumour size was $46 \mathrm{~mm}$ (range from 4 to $295 \mathrm{~mm}$ ). The median mitotic index was 4 per $50 \mathrm{hpf}$ (range from 0 to 45 per $50 \mathrm{hpf}$ ). Risk stratification according to Miettinen classified the patients into benign 5 (9.6\%), very low risk in $9(17.3 \%)$, low risk $13(25.0 \%)$, intermediate risk $10(19.2 \%)$ and high risk in 13 patients (25.0\%). Two patients were unable to be classified. Characteristics are shown in Table 1. 
Table 2 Treatment parameters

\begin{tabular}{lc}
\hline Operations & $\mathrm{N}(\%)$ \\
\hline Gastric wedge resection & $17[33]$ \\
Partial gastrectomy & $5[10]$ \\
Small bowel resection & $11[21]$ \\
Multivisceral resection & $11[21]$ \\
Other procedure & $8[15]$ \\
Resection & \\
R0 & $46[88]$ \\
0 & $4[8]$ \\
0 & $2[4]$ \\
\hline
\end{tabular}

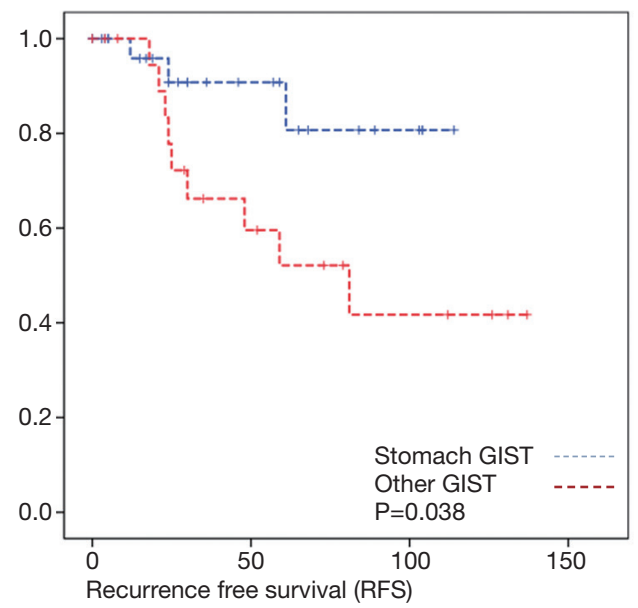

Figure 1 The RFS comparing gastric GIST (blue dashed lines) with GIST other origin (red dashed lines) (n=52). GIST, gastrointestinal stromal tumors; RFS, recurrence-free survival.

\section{Treatment and surgical outcome}

Overall, 3 patients were subjected to neoadjuvant imatinib therapy because of tumor size with the aim to reach resectability. Duration of neoadjuvant therapy was 4 months with partial response to treatment in all 3 patients.

Surgical treatment was performed per laparoscopy in $3(6 \%)$ and laparotomy in patients 49 (94\%). Performed surgical procedures are shown in Table 2. Complete surgical resection could be achieved in 46 patients $(88 \%)$.

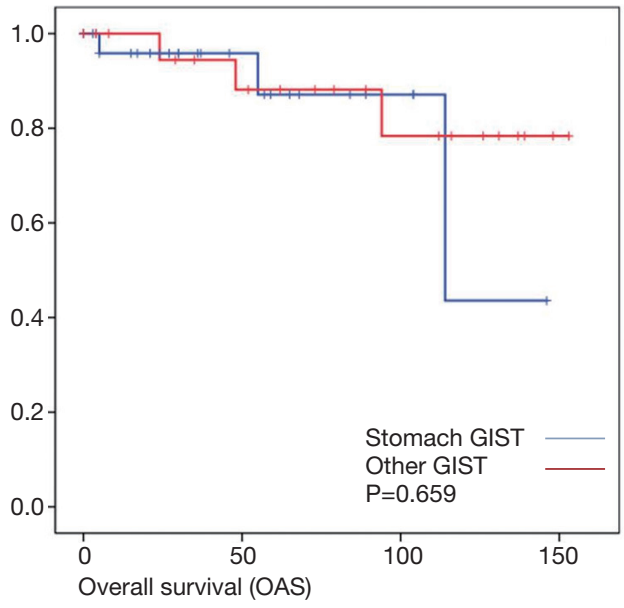

Figure 2 The OAS comparing gastric GIST (blue solid lines) and non-gastric GIST (red solid lines) ( $\mathrm{n}=49)$. GIST, gastrointestinal stromal tumors; OAS, overall survival.

\section{Adjuvant treatment and clinical course}

Twenty-one patients (40.4\%) of the study population received imatinib chemotherapy in the adjuvant setting in addition to 3 patients in the neoadjuvant setting (5.7\%). Duration of adjuvant therapy with imatinib was 42 months (range 4 to 60 months).

\section{Survival and relapse patterns}

\section{Oncologic outcome}

Follow-up data were available for 48 patients. Recurrence was seen in 12 patients ( $25 \%)$. Median follow-up was 50 months. Mean recurrence free survival was 45.6 months (range, 36-57 months). Mean overall survival was 58.9 months (range, 46-73 months). Median recurrence free survival measured from time of surgery was 30 months. One, two-, three- and five-year recurrence-free survival was $98 \%, 85 \%$, $81 \%$ and $77 \%$.

Overall survival was calculated for 46 patients. Three patients had to be excluded for having terminal stage oncologic disease of another leading entity on GISTdiagnosis. For 2 other patients no follow-up data were available. Median overall survival rate was 52 months during the study period. One, two-, three- and five-year overall survival was $98 \%, 96 \%, 96 \%$ and $91 \%$ respectively (Figures 1-6). 


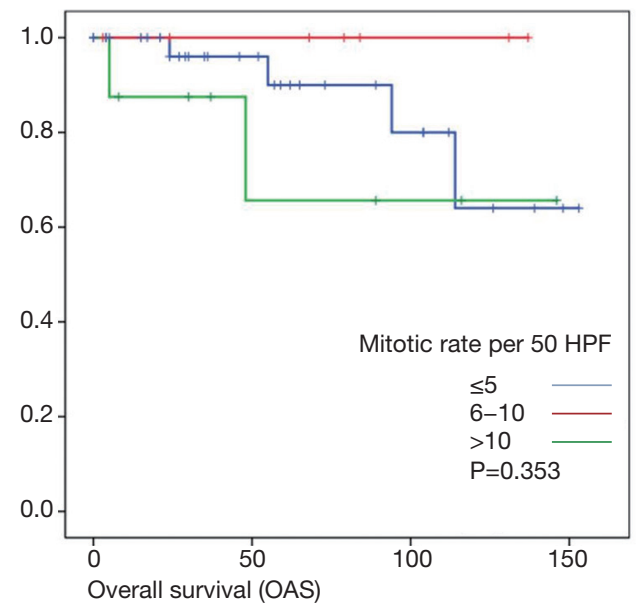

Figure 3 OAS of all patients divided into 3 groups by the mitotic rate $(n=49)$. OAS, overall survival.

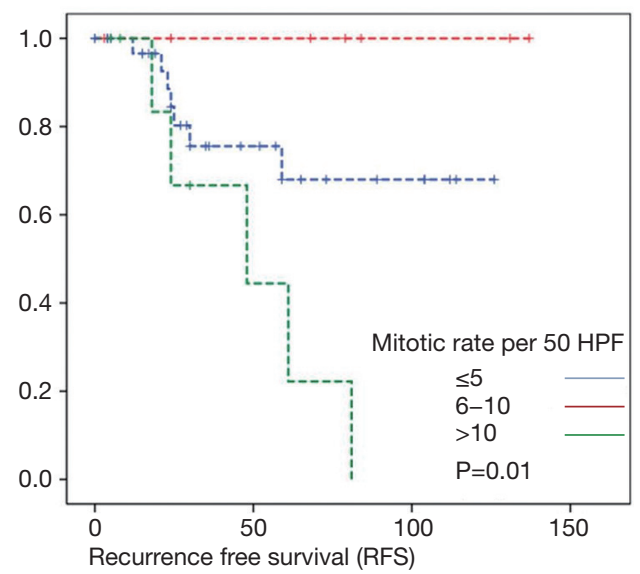

Figure 4 Compares the RFS of all patients divided by their mitotic rate $(\mathrm{n}=52)$. RFS, recurrence-free survival.

\section{Discussion}

Surgical resection remains the cornerstone in early primary and locally advanced or recurrent GIST. Integration and timing of systemic tyrosine kinase inhibitors is the additional decisive factor for overall and recurrence free survival. In this retrospective evaluation, we investigated the outcome of patients with surgical resection and adjuvant or neoadjuvant chemotherapy with imatinib. We identified the outcome of the personalized medical and operative treatment concepts of patients with GISTs. We analyzed the influence of the mitotic activity measured with Ki-67.

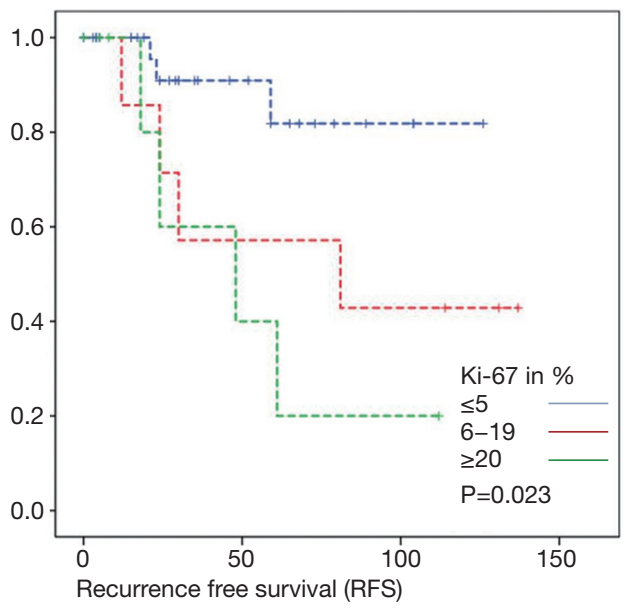

Figure 5 Compares the RFS of all patients divided by their Ki67/ MiB1 expression. The RFS is worst for the patients with high Ki67 expression $>20 \%$ and best for Ki67 expression $<5 \%(n=48)$. RFS, recurrence-free survival.

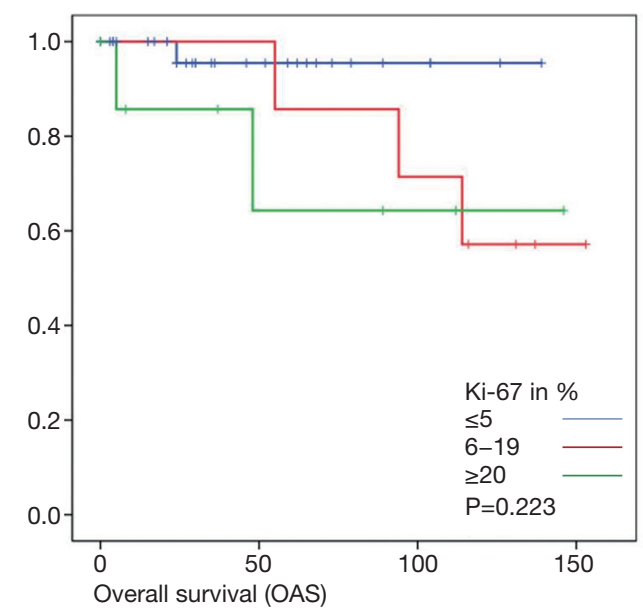

Figure 6 Compares the OAS of all patients divided by their Ki67/ MiB1 expression. The OAS is independent of Ki67 expression $(n=48)$. OAS, overall survival.

Surgical removal with complete excision and without tumor rupture is the only curative therapy and mainstay for localized primary resectable GISTs (5). In the evaluated time period we performed most of the resections within an open operation. For surgical resection less invasive methods are increasingly favoured supported by actual studies showing superiority in postoperative outcome without compromising oncologic outcome $(6,7)$. Independent from type of operation (open, minimally invasive or minimally- 
invasive assisted) it is in this context still and always to remember that safe oncologic resection is primary concern avoiding incomplete resection or tumor rupture caused due to inexperience of surgeon or access of surgery. Goal of surgery was to remove the tumor without tumor rupture. In our study $88 \%$ of the patients were completely resectable, $8 \%$ were with marginal resection and $4 \%$ were with macroscopic incomplete resection. In this respect 3- and 5 -year recurrence-free survival of $81 \%$ and $77 \%$ are good results.

The evaluation of tumor location and long-term oncologic outcome showed in our evaluation no significant differences regarding overall survival depending on the overall very low mortality rate. Regarding recurrence free survival there was a significant difference between gastric and the other tumor locations.

In our study chemotherapy was indicated in $46 \%$ of patients. Median duration of treatment was 42 months. Regarding duration of chemotherapy, the PERSIST-5 phase II study investigated the extending of postoperative treatment with imatinib for 5 years in patients with high risk of recurrence confirming imatinib effectiveness (8).

As most of the studied patients were alive or died from another disease, the overall survival was not influenced regarding tumor location or mitotic index classified in Miettinen or Ki-67\%. Indeed, the recurrent free survival was significantly influenced by tumor location. Best prognosis was seen in gastric tumor location compared to the other tumor locations $(\mathrm{P}=0.038)$. The mitotic index analyzed in the Miettinen classification showed best recurrence free survival with mitotic count between 6 and $10 \mathrm{HPF}$. The proliferation marker Ki-67 better predicted recurrence free survival. Best recurrence free survival was seen in patients of the lowest proliferation activity of fewer than $5 \%(\mathrm{P}=0.023)$. As clinical behavior and oncologic outcome is different even for patients within the same risk-stratification GIST group, further aspects have to be evaluated. In our evaluation the correlation between Ki67 was the best predictor of recurrence free survival. This is in the line with earlier publications (9).

\section{Conclusions}

Complete resection remains therapy of choice. Due to rarity and heterogeneity of this pathology, individualization of treatment is necessary in each specific case. Imatinib is indicated in locally advanced GIST. The role of Ki-
67 labeling index as a predictor of outcome has to further evaluated.

\section{Acknowledgements}

None.

\section{Footnote}

Conflicts of Interest: The authors have no conflicts of interest to declare.

Ethical Statement: Ethical committee approval was given for the retrospective design (No. 551/2015BO2). Consent to participate is not possible because of the design of the study without intervention.

\section{References}

1. Corless CL, Fletcher JA, Heinrich MC. Biology of gastrointestinal stromal tumors. J Clin Oncol 2004:22:3813-25.

2. Shinomura Y, Kinoshita K, Tsutsui S, et al. Pathophysiology, diagnosis, and treatment of gastrointestinal stromal tumors. J Gastroenterol 2005;40:775-80.

3. Blay JY, Bonvalot S, Casali P, et al. Consensus meeting for the management of gastrointestinal stromal tumors. Report of the GIST Consensus Conference of 20-21 March 2004, under the auspices of ESMO. Ann Oncol 2005;16:566-78.

4. Dematteo RP, Ballman KV, Antonescu CR, et al. Adjuvant imatinib mesylate after resection of localised, primary gastrointestinal stromal tumour: a randomised, double-blind, placebo-controlled trial. Lancet 2009;373:1097-104.

5. Miettinen M, Lasota J. Gastrointestinal stromal tumors: pathology and prognosis at different sites. Semin Diagn Pathol 2006;23:70-83.

6. Melstrom LG, Phillips JD, Bentrem DJ, et al. Laparoscopic versus open resection of gastric gastrointestinal stromal tumors. Am J Clin Oncol 2012;35:451-4.

7. Ye $\mathrm{L}, \mathrm{Wu} \mathrm{X}, \mathrm{Wu}$ T, et al. Meta-analysis of laparoscopic vs. open resection of gastric gastrointestinal stromal tumors. PLoS One 2017;12:e0177193.

8. Raut CP, Espat J, Maki RG, et al. PERSIST-5: five year extended treatment with adjuvant imatinib for patients 
with intermediate/high risk primary gastrointestinal stromal tumor (GIST). Available online: https:// meetinglibrary.asco.org/record/152120/slide

9. Sugita S, Hirano H, Hatanaka Y, et al. Image analysis

doi: $10.21037 / \operatorname{tgh} .2018 .12 .02$

Cite this article as: Bachmann R, Strohäker J, Kraume J, Königsrainer A, Ladurner R. Surgical treatment of gastrointestinal stromal tumours combined with imatinib treatment: a retrospective cohort analysis. Transl Gastroenterol Hepatol 2018;3:108. is an excellent tool for quantifying $\mathrm{Ki}-67$ to predict the prognosis of gastrointestinal stromal tumor patients. Pathol Int 2018;68:7-11. 